\title{
FATORES SOCIODEMOGRÁFICOS E DE SAÚDE DE PESSOAS COM TUBERCULOSE EM TRÊS CIDADES NO SUL DO BRASIL
}

\section{SOCIODEMOGRAPHIC AND HEALTH FACTORS OF PEOPLE WITH TUBERCULOSIS IN THREE CITIES IN SOUTHERN BRAZIL}

\section{FACTORES SOCIODEMOGRÁFICOS Y DE SALUD DE LAS PERSONAS CON TUBERCULOSIS EN TRES CIUDADES DEL SUR DE BRASIL}

\author{
Sabrina da Silva de Souza ${ }^{1}$, Julia Estela Willrich Boell ${ }^{2}$, Samara Eliane Rabelo Suplici ${ }^{3}$, Katheri Maris Zamprogna ${ }^{4}$
}

\section{RESUMO}

Objetivo: Descrever as características sociodemográficas de pessoas acometidas por tuberculose, de resposta ao tratamento e uso de substâncias nocivas à saúde, em três municípios prioritários no controle da doença, no Sul do Brasil.Método:Estudo transversal,descritivo-exploratório, realizado com 209 pessoas em tratamento de tuberculose. Aplicou-se instrumento para coletar dados sociodemográficose de saúde dos participantes. Realizada análise descritiva dos dados. Resultados: Os participantes possuíam em média, 44 anos (DP:16,1); em sua maioria solteiros (50,7\%), com crença religiosa (91\%), ativos economicamente (61,7\%), com ensino fundamental (65\%), renda média de 1.791,91 reais. Entre os participantes, $28,2 \%$ eram fumantes, enquanto $43,5 \%$ nunca fumaram; $53,4 \%$ negaram etilismo e $46,5 \%$ afirmaram o uso. Dos participantes, $56 \%$ eram de casos novos da doença, enquanto $7,7 \%$ apresentavam recidiva. Houve $78,5 \%$ que apresentaram cura ao final do tratamento e $3,8 \%$, cujo tratamento não obteve resposta satisfatória, sendo 1,9\%, aqueles que evoluíram para óbito. Conclusão: Analisar o perfil das pessoas atendidas nessas cidades permitiu maior compreensão acerca da tuberculose, ampliando o olhar sobre o tratamento e acompanhamento de tais pacientes, bem como dos determinantes que estão intimamente relacionados ao curso da doença.

Descritores: Cooperação e Adesão ao Tratamento; Atenção à Saúde; Tuberculose.

\section{ABSTRACT}

Objective: to describe the sociodemographic characteristics, response to treatment and use of harmful substances of people with tuberculosis from three priority municipalities in the control of the disease in southern Brazil. Method: cross-sectional, descriptive and exploratory study, conducted with 209 people undergoing treatment for tuberculosis. An instrument was applied to collect sociodemographic and health data from the participants. The descriptive analysis of the data was performed. Results: the participants were on average 44 years old (SD: 16.1); mostly single (50.7\%), with religious beliefs (91\%), economically active $(61.7 \%)$, with elementary education (65\%), average income of $R \$ 1,791.91$. Of the participants, $28.2 \%$ were smokers, while $43.5 \%$ never smoked; $53.4 \%$ denied alcoholism and $46.5 \%$ stated the use. Of the participants, $56 \%$ were new cases of the disease, while $7.7 \%$ had relapses. A total of $78.5 \%$ of the participants presented cure at the end of the treatment and $3.8 \%$ did not obtain a satisfactory result with the treatment, in which $1.9 \%$ died. Conclusion: the analysis of the profile of people treated in these cities allowed a greater understanding about tuberculosis, broadening the view on the treatment and the follow-up of such patients, as well as the determinants that are closely related to the course of the disease.

Descriptors: Treatment Adherence and Compliance; Health Care; Tuberculosis

\section{RESUMEN}

Objetivo: Describir las características sociodemográficas de las personas con tuberculosis, la respuesta al tratamiento y el uso de sustancias nocivas en tres municipios prioritarios para el control de la enfermedad en el sur de Brasil. Método: Estudio transversal, descriptivo y exploratorio, realizado con 209 personas con tratamiento por tuberculosis. Se aplicó un instrumento para recopilar datos sociodemográficos y de salud de los participantes. Se realizó un análisis descriptivo de los datos. Resultados: Los participantes tenían un promedio de 44 años (DE: 16,1); en su mayoría solteros (50.7\%), con creencias religiosas (91\%), económicamente activos (61.7\%), con educación primaria (65\%), ingreso medio de $1,791.91$ reales. De los participantes, $28.2 \%$ eran fumadores, mientras que $43.5 \%$ nunca fumaron; El 53.4\% negó el alcoholismo y el $46.5 \%$ declaró el uso. De los participantes, el $56 \%$ eran casos nuevos de la enfermedad, mientras que el $7,7 \%$ tenía recaídas. Hubo $78.5 \%$ que presentaron cura al final del tratamiento y $3.8 \%$ cuyo tratamiento no obtuvo una respuesta satisfactoria, de estos $1.9 \%$ murieron. Conclusión: El análisis del perfil de las personas tratadas en estas ciudades permitió una mayor comprensión sobre la tuberculosis, ampliando la visión sobre el tratamiento y el seguimiento de dichos pacientes, así como los determinantes que están estrechamente relacionados con el curso de la enfermedad.

Descriptores: Cumplimiento y Adherencia al Tratamiento; Atención a la Salud; Tuberculosis.

'Doutora em Enfermagem. Enfermeira da emergência adulto do Hospital Universitário da Universidade Federal de Santa Catarina. ${ }^{2}$ Doutora em enfermagem pela Universidade Federal de Santa Catarina. Professora da Faculdade Santa Catarina. ${ }^{3}$ Doutora em enfermagem pela Universidade Federal de Santa Catarina. Professora da Faculdade Estácio de Sá. ${ }^{4}$ Doutorando pelo Programa de Pós Graduação em Enfermagem da Universidade Federal de Santa Catarina. 


\section{INTRODUÇÃO}

A saúde resulta da interação entre aspectos biológicos, mentais, sociais, culturais, econômicos e sua relação com o meio ambiente e a sociedade. Nesse sentido e, atendendo ao chamado realizado pela OMS aos profissionais de saúde sobre a necessidade de priorizar a dimensão humana, é necessário entender o indivíduo, conhecer seu contexto, a realidade em que ele vive e os sofrimentos e dificuldades que enfrenta ${ }^{(1)}$.

Dentre as doenças que ainda representam um desafio para o sistema de saúde está a tuberculose (TB). Tanto a TB quanto a Síndrome da Imunodeficência Adquirida (AIDS) são apontadas como meta para o cumprimento dos objetivos do desenvolvimento sustentável, que é promover o bem-estar para todos, em todas as idades, sendo apontado para essas duas doenças a sua eliminação até o ano de 2030. Para que tal feito ocorra, é necessário cuidado integrado, centrado no paciente e prevenção; políticas arrojadas e sistemas de apoio; intensificação das pesquisas e inovação. A TB é a doença que mais emblematicamente caracteriza a determinação social da pobreza no processo saúde/doença de uma população(2).

As taxas de incidência, prevalência e mortalidade evidenciam a magnitude do problema. A TB é uma doença com alta incidência mundial sendo, anualmente, descobertos milhões de casos novos e ainda, considerada entre as doenças infecciosas, a de maior mortalidade ${ }^{(3)}$. Foram registrados 72.788 casos novos de TB em 2018. Em 2017, foram registrados 4.534 óbitos pela doença, o que equivale a um coeficiente de mortalidade de 2,2 óbitos/100 mil habitantes ${ }^{(4)}$.

O Brasil, ocupa a 20a posição entre os 30 países com maior carga de TB, considerados prioritários para o controle da doença, detêm $87 \%$ do total de casos mundiais ${ }^{(5)}$ : em 2016 foram notificados 58.601 casos. Em Santa Catarina, os registros indicam que naquele ano foram notificados 1.585 casos novos de TB, sendo que 1.046 pessoas foram curadas com o tratamento adequado. Do total de casos, 1.159 foram confirmados como TB pulmonar ${ }^{(6)}$.

A cobertura universal é um elementochave para o alcance dos objetivos globais (ODS), ao exigir que os governos assumam a responsabilidade de garantir o acesso universal aos serviços de saúde, promovendo a eliminação da pobreza, a equidade, a justiça e os direitos humanos. O cumprimento de suas metas é fundamental para que se proporcionem melhores serviços às populações mais vulneráveis, contribuindo, assim, com o enfrentamento da $\mathrm{TB}^{(7)}$.

Diagnosticar e tratar corretamente os casos de TB pulmonar são medidas fundamentais para o seu controle. Esforços têm sido realizados para que a doença seja diagnosticada precocemente e o paciente inicie o tratamento adequado, de forma que a cadeia de transmissão do bacilo seja interrompida ${ }^{(8)}$.

Isso evidencia o quão importante é o tratamento da TB, pois é a principal forma de diminuir a mortalidade. No entanto, a adesão ao tratamento representa um desafio para o sistema de saúde. Isso porque os conhecimentos, atitudes e percepções das pessoas afetadas pela TB, podem favorecer a adesão ou não adesão ao tratamento ${ }^{(9)} . A$ realização irregular do tratamento da TB contribui para a resistência medicamentosa, além de manter a transmissão da doença ${ }^{(10-11)}$.

Por maior que seja o enfoque no tratamento, muitas são as disparidades (regionais e nacionais) que ocorrem, desde o início da elucidação da doença (sintomas e diagnóstico) até $\mathrm{o}$ desfecho final do caso. $O$ tratamento completo é indispensável para o controle da TB, além da necessária reorganização do sistema de saúde, visando a resultados em termos de diagnóstico precoce, tratamento padronizado, oferta gratuita de medicamentos e encerramento dos casos por alta/cura ${ }^{(12)}$.

Fatores socioeconômicos relacionados à doença $^{(7)}$ apresentam atuação direta no surgimento de casos novos, como a baixa escolaridade ou a renda. Dessa forma, em face da relevância da temática, no cenário mundial, é necessário conhecer o perfil das pessoas, como se comporta a doença em relação a alguns fatores, como etilismo, tabagismo, situação econômica bem como condições de saúde e determinantes que estejam intensificando, diminuindo ou, por vezes, mantendo o curso da doença. Dessa forma, conduziu-se, este estudo,com o objetivo de descrever as condições de saúde e características socioeconômicas das pessoas acometidas por TB, em três municípios prioritários no controle da doença, no Sul do Brasil.

\section{MÉTODO}

Estudo transversal, descritivo-exploratório desenvolvido em três serviços de saúde que 
acompanham pessoas com TB de três municípios, no controle dessa doença, em Santa Catarina. De acordo com o Programa Nacional de Controle da Tuberculose (PNCT), entre os indicadores para classificar os municípios como prioritários para TB estão: ser uma capital; municípios de região metropolitana de capitais com mais de 30 casos, na média, ou maior valor notificado dos últimos três anos; municípios com número de habitantes menor que 100 mil e com carga de TB igual ou superior a 30 casos; taxa de incidência superior a $80 \%$ da taxa do país ou taxa de mortalidade superior a do país ${ }^{(13)}$.

Assim, a escolha dos três municípios ocorreu, em decorrência da semelhança na forma de organização desses municípios, na atenção à pessoa com TB e por serem prioritários no controle da doença no país. O principal modelo de assistência dos três municípios investigados é a Estratégia Saúde da Família (ESF), com a equipe formada por médico, enfermeiros, agentes comunitários de saúde e técnicos de enfermagem. Nesses três municípios há um programa municipal de controle da TB, no qual as pessoas são acompanhadas, mensalmente, com consultas médicas e ações de enfermagem. Fizeram parte do estudo, 209 pessoas que estavam em tratamento para TB, a amostra contemplou todas as pessoas que estavam em tratamento para TB nos referidos municípios e que aceitaram participar da pesquisa. A coleta de dados ocorreu, no período de outubro de 2015 a março de 2018, o longo período de coleta se deu, em virtude da dificuldade de acessar todos os pacientes em tratamento nos referido municípios e de encontrar profissionais para coletar os dados. As entrevistas foram realizadas, por meio da aplicação de questionário, por sete enfermeiras, previamente treinadas. Para a seleção dos participantes, foram adotados os seguintes critérios de inclusão: idade acima de 18 anos; ter capacidade de se comunicar a partir de avaliação subjetiva do pesquisador e estar em tratamento nos serviços de saúde no período da coleta de dados.

A coleta de dados foi realizada, utilizandose questionário, contendo informações sociodemográficas(gênero, raça, religião, situação conjugal, renda, ativo no trabalho)e de condições de saúde (uso de tabaco e álcool, a tuberculose e a fase do tratamento, a adesão ao tratamento da TB).Em determinadas variáveis, nem todos os participantes responderam às questões, dessa forma, na apresentação dos resultados, nas tabelas foi exposto entre parênteses, ao lado daquelas variáveis o ( $n$ ) de respostas, quando não alcançados os 209 participantes.

Os dados foram armazenados no programa Excel ${ }^{\circledR}$ da Microsoft ${ }^{\circledR}$. A análise dos dados foi efetuada por meio do SPSS (IBM SPSS versão 22), utilizando a estatística descritiva para as variáveis quantitativas (média, desvio padrão (DP), valores máximos e mínimos). Foram respeitados todos os preceitos éticos determinados pela Resolução $n$ o 466/2012 do Conselho Nacional de Saúde do Ministério da Saúde. O estudo recebeu parecer favorável, em 07 de dezembro de 2015, pelo Comitê de Ética da instituição hospitalar da Universidade Federal de Santa Catarina (UFSC) e pelo Comitê de Ética em Pesquisas com Seres Humanos da UFSC, sob o protocolo de número 1.249.257.

\section{RESULTADOS E DISCUSSÃO}

Participaram do estudo 209 pessoas, sendo $115(55 \%)$ do município de Joinville, $57(27,3 \%)$ de São José e $37(17,7 \%)$ de Criciúma. Os participantes do estudo possuíam, em média, 44 anos de idade (DP: 16,1). Em relação ao gênero, participaram 140 (67\%) homens e 69 (33\%) mulheres. A renda individual apresentou média de $R \$ 1.791,91$ reais (DP:58\%). As características sociodemográficas (Tabela 1) e as condições de saúde (Tabela 2) estão demonstradas, a seguir.

O predomínio de pessoas do sexo masculino com TB encontrado, neste estudo, corrobora com os dados de outro estudo, ${ }^{(14)}$ no qual foram encontradas taxas semelhantes tanto no sexo feminino $(31,1 \%)$ quanto no masculino $(68,9 \%)$. Esses valores também estão próximos àqueles encontrados em pesquisa realizada na assistência hospitalar (30,1 e 69,9\%) e na atenção primária (33,8 e 66,2\%), da cidade de São Paulo $^{(15)}$ e também,em estudo realizado para verificar o perfil dos pacientes com TB em outro estado Belo Horizonte, Minas Gerais(35,2 e $64,8 \%)^{(16)}$. Como a TB está relacionada à má alimentação, falta de higiene, tabagismo, alcoolismo ou qualquer outro fator que gere baixa resistência orgânica ${ }^{(17)}$, a combinação desses fatores, na população masculina, pode ser a responsável pela diferença entre homens e mulheres. Distinções comportamentais e culturais entre homens e mulheres podem estar relacionadas a essa diferença ${ }^{(18)}$.

Cabe salientar que, nos países em desenvolvimento, $\quad 80 \%$ dos infectados 
encontram-se entre 15 e 59 anos $^{(8,14)}$; estão, portanto, na faixa de maior produtividade social. Uma das possíveis explicações para a elevada frequência da TB em adultos jovens, relaciona-se aos hábitos adotados por estes, deixando-os mais expostos à doença ${ }^{(19)}$ ao aumento da prevalência do HIV e ao alcoolismo ${ }^{(20)}$.A TB afeta, principalmente, a população economicamente ativa, sobretudo os homens em idade produtiva,acarretando retardo do crescimento econômico,com prejuízo no desenvolvimento da sociedade,gerando mais pobreza e exclusão social $^{(17)}$. A média de 44 anos, obtida no presente estudo se assemelha a estudo realizado, na cidade de São Paulo, o qual apresentou na faixa etária de 20 a 59 anos $^{(15)}$ a maioria dos casos de TB, e também, a outro estudo que apresentou maior taxa de incidência entre 40 a 59 anos $^{(17)}$.

Com relação à raça, os dados obtidos no presente estudo que evidenciam maior prevalência de TB na população branca (74,2\%) divergem do estudo de Ferraz ${ }^{(14)}$ que encontrou maior prevalência entre a população negra $(76,7 \%)$, tal achado decorre, pois nos municípios estudados há predominância da população branca, pelo censo IBGE 2010, 84\% da população de SC se declarara branca(21-22).

Tabela 1 - Características sociodemográficas de pessoas com tuberculose em três municípios prioritários no controle da doença no sul do Brasil. São José - SC, Brasil, 2015 a 2018.

\begin{tabular}{|c|c|c|}
\hline Variável & $\mathbf{N}$ & $\%$ \\
\hline \multicolumn{3}{|l|}{ Gênero } \\
\hline Feminino & 69 & 33,0 \\
\hline Masculino & 140 & 67,0 \\
\hline \multicolumn{3}{|l|}{ Raça } \\
\hline Branco & 155 & 74,2 \\
\hline Negro & 27 & 12,9 \\
\hline Pardo & 27 & 12,9 \\
\hline \multicolumn{3}{|l|}{ Religião (n=208) } \\
\hline Sem religião & 19 & 9,2 \\
\hline Com religião & 189 & 90,8 \\
\hline \multicolumn{3}{|l|}{ Situação Conjugal (n=208) } \\
\hline Casado & 69 & 33,2 \\
\hline Viúvo & 9 & 4,3 \\
\hline Divorciado/Separado & 24 & 11,5 \\
\hline Solteiro & 106 & 51,0 \\
\hline \multicolumn{3}{|l|}{ AtivoEconomicamente } \\
\hline Não trabalha & 78 & 37,3 \\
\hline Trabalha & 129 & 61,7 \\
\hline Não responderam & 2 & 1,0 \\
\hline
\end{tabular}

Fonte: Elaborada pelas autoras combase nos dados coletados.

O hábito do uso do tabaco foi questionado aos participantes, sendo que, a maioria, relatou que nunca fumara $(43,8 \%) ; 58$ participantes $(27,8 \%)$ relataram ter fumado, porém já cessaram o hábito, e 59 participantes (28,4\%)são fumantes.Quanto à variável etilismo, $111(53,1 \%)$ participantes negaram o uso e 97 (46,5\%) afirmaram a ingestão de álcool, conforme Tabela 2. O tabagismo, fator que altera a atividade do sistema imunológico, repercurte diretamente sobre o curso da doença, uma vez que sua administração leva à diminuição do sistema de 
defesa do organismo, predispõe o paciente a outras comorbidades ${ }^{(17)}$. Quanto ao uso de álcool, não chega a ser muito diferente, dado que estudo realizado mostrou probabilidade 24 vezes maior dos pacientes que fazem uso da substância, estarem propensos a outras enfermidades ${ }^{(17)}$.

Tabela 2 - Tabagismo, consumo de álcool e frequência de uso do álcool em pessoas com tuberculose em três municípios prioritários no controle da doença, no sul do Brasil. São José - SC, Brasil, 2015 a 2018.

\begin{tabular}{lll}
\hline \hline Variável & N & $\%$ \\
\hline Tabagismo & 91 & 43,7 \\
Nunca Fumou & 58 & 27,9 \\
Já fumou, mas parou & 59 & 28,4 \\
Fuma & & \\
Consumo de álcool & 97 & 46,6 \\
Sim & 111 & 53,4 \\
Não & & \\
Frequência consumo de álcool & & 46,4 \\
2 a 3 dias por semana & 45 & 6,2 \\
4 a 6 dias por semana & 6 & 18,6 \\
Todos os dias & 18 & 14,4 \\
Uma vez por mês & 14 & 2,0 \\
Duas a quatro vezes por mês & 2 & 12,4 \\
Esporadicamente ao ano & 12 & \\
& & 35,0 \\
Frequência consumo de álcool em anos & & 23,8 \\
$<5$ anos & 34 & 17,5 \\
6 a 10 anos & 34,4 \\
11 a 20 anos & 23 & 11,3 \\
$>30$ a 30 anos & 17 & \\
& 12 & \\
\hline \hline
\end{tabular}

Fonte: Elaborada pelas autoras combase nos dados coletados.

Dos participantes que relataram consumir bebida alcoólica, $34(35,0 \%)$ relataram etilismo, por menos de cinco anos, sendo $23(23,8 \%)$ aqueles que ingeriram álcool de seis a dez anos, $17(17,5 \%)$ os que ingeriram de onze a vinte anos e, o menor quantitativo de $12 \quad(12,4 \%)$ participantes, que relataram a ingesta, de 21 a 30 anos, seguido daqueles que bebem há mais de 30 anos, com um quantitativo de 11 (11,3\%) participantes.

Chama a atenção que, entre as variáveis, a frequência de consumo alcoólico esteve presente de 2 a 3 dias na semana, referido por $45(46,4 \%)$ participantes, sendo $18(18,6 \%)$ os que bebem todos os dias; seguidos de $14(14,4 \%)$ que bebem uma vez ao mês e, finalmente, dois $(2,1 \%)$ que bebem de duas a quatro vezes ao mês e 12 participantes $(12,4 \%)$ realizam a ingesta alcoólica, esporadicamente, no ano (Tabela 2 ).

Se considerarmos que a TB é doença vinculada a fatores de pobreza, condições de vida e de trabalho e que o uso de álcool gera deterioração do sistema imunológico, esse pode ser considerado fator de predisposição do paciente à infecção tuberculosa ${ }^{(17)}$. Em se tratando do tabaco, o mesmo também está relacionado a maior chance de propensão a outras enfermidades, sendo possível inferir que os pacientes em uso de ambas as substâncias estarão mais propensos à piora do prognóstico, dado ser esse um processo infecioso que, em condições de tratamento correto e de estilo e hábitos de vida saudáveis apresenta melhora importante no decurso da doença ${ }^{(17)}$.

Entre os participantes foram registrados que a maioria tratava-se de casos novos, sendo 116 (55\%), no entanto, ainda foram constatadas pessoas apresentando recidiva da doença, sendo $16(7,7 \%)$ como se mostra na Tabela 3.Observa-se que o percentual de transferência é elevado, o que pode estar relacionado ao diagnóstico realizado em hospitais, sendo transferidos, posteriormente, para a atenção primária dos municípios, assim como a mudança de domicílio 
dessas pessoas. Parece que essas transferências têm ocorrido de forma sistemática, mantendo o monitoramento do paciente pela vigilância epidemiológica, evitando o contágio de outras pessoas e assegurando que o mesmo receba tratamento adequado, durante sua movimentação nos diversos pontos de atenção da saúde, informando a unidade de destino sobre a transferência.

Tabela 3 -Características da doença quanto aos casos novos, transferência, reingresso e recidiva em três municípios prioritários no controle da doença no sul do Brasil. São José - SC, Brasil, 2015 a 2018.

\begin{tabular}{lll}
\hline \hline \multicolumn{1}{c}{ Variável } & $\mathbf{n}$ & $\%$ \\
\hline Caso novo & 116 & 55,8 \\
Transferência & 66 & 31,7 \\
Reingresso após abandono & 10 & 4,8 \\
Recidiva da TB & 16 & 7,7 \\
\hline \hline
\end{tabular}

Fonte: Elaborada pelas autoras com base nos dados coletados

Entre os participantes, foram registrados que 164 (78,5\%) obtiveram resposta favorável ao tratamento, tendo cura ao final do mesmo, dez $(4,8 \%)$ abandonaram o tratamento, quatro $(1,9 \%)$ evoluíram para óbito, 18 (8,6\%) precisaram transferir seu tratamento para outro município, oito $(3,8 \%)$ o tratamento não obteve resposta satisfatória, sendo considerado falência do tratamento e cinco $(2,4 \%)$ evoluíram para TB multidroga resistência (TBMR) como se mostra na Tabela 4.

Tabela 4 - Resposta ao tratamento à tuberculose em três municípios prioritários no controle da doença no sul do Brasil. São José - SC, Brasil, 2015 a 2018.

\begin{tabular}{lcc}
\hline \hline Variável & $\mathbf{N}$ & $\%$ \\
\hline Cura & 164 & 78,5 \\
Abandono & 10 & 4,8 \\
Óbito & 4 & 1,9 \\
Transferência & 18 & 8,6 \\
Falência & 8 & 3,8 \\
TBMR & 5 & 2,4 \\
\hline \hline
\end{tabular}

Fonte: Elaborada pelas autoras combase nos dados coletados.

Quanto à resposta ao tratamento, o percentual de cura alcançou $78,5 \%$, apesar de maior que outros estudos ${ }^{(14,20)}$, ainda é inferior à meta proposta pelo MS, que é de curar $85 \%$ dos casos diagnosticados. Esse indicador é de grande utilidade para subsidiar a avaliação da efetividade do tratamento ${ }^{(21)}$.O percentual de abandono foi de $4,8 \%$, por sua vez, obedece a meta traçada pelo $\mathrm{MS}$, que é de $5 \%$ e bem abaixo do observado nas regiões brasileiras. No Brasil, esse percentual apresenta-se variável nas suas diversas regiões ${ }^{(21)}$, sendo que na região norte fica em torno de $9,8 \%$, no nordeste $9,5 \%$, no sudeste $11,8 \%$, no centrooeste $11,2 \%$ e no sul $10 \%{ }^{(4)}$.

O abandono do tratamento ainda é um grande desafio entre as pessoas com TB e suas múltiplas causas estão relacionadas às questões individuais, sociais e ao sistema de saúde.

Quanto aos aspectos individuais, o uso de drogas, a ocorrência de outras doenças, 
principalmente as crônicas são identificadas como contribuindo para a não adesão ao tratamento ${ }^{(22)}$.

\section{CONCLUSÃO}

Analisar as condições de saúde e características sociodemográficas das pessoas que possuem TB permitiu maior compreensão acerca dos fatores e determinantes associados à doença, bem como o perfil das pessoas e suas condições de saúde, especialmente nas três cidades estratégicas de Santa Catarina.

A importância de conhecer características, como: gênero, raça, situação socioeconômica e hábitos de vida como tabagismo e etilismo, mostraram-se intimamente relacionados ao melhor prognóstico ou não da doença. Com isso, tem-se a representação do cenário da TB e de seus fatores relacionados, possibilitando a intensificação de ações, nessas populações, que contribuam à diminuição da transmissão, a partir da melhoria da adesão ao tratamento, reduzindo, assim, as taxas de abandono e o surgimento de cepas resistentes, dado que tais condições e características são aspectos importantes para o seu controle, refletem a permanência da doença como desafio mundial.

\section{REFERÊNCIAS}

1- World Health Organization (WHO). The world health report 2008: Primary health care now more than ever. Washington: WHO; 2008.

2- Brasil, Ministério da Saúde. Política Nacional de Atenção Básica. Brasília: Ministério da Saúde; 2012.

3- Brasil, Ministério da Saúde. Tuberculose no mundo. Brasília: Ministério da Saúde; 2016.

4- Brasil, Ministério da Saúde. Brasil livre da tuberculose: Evolução dos cenários epidemiológicos e operacionais da doença. Bol Epidemiol. 2019 [citado em 2 dez 2018]; 50(9):118. Disponível em: https://portalarquivos2.saude.gov.br/images/pdf 2019/marco/22/2019-009.pdf

5- Brasil, Ministério da Saúde. Guia de vigilância em saúde. Brasília: Ministério da Saúde; 2019.

6- Brasil, Ministério da Saúde. Tuberculose Casos confirmados notificados no Sistema de Informação de Agravos de Notificação - Santa Catarina. Tabnet 2016 [citado em 01 jul 2019]. Disponível em: http://tabnet.datasus.gov.br/cgi/tabcgi.exe?sina nnet/cnv/tubercsc.def

7- Barreira D. Os desafios para a eliminação da tuberculose no Brasil. Epidemiol Serv Saúde 2018;27(1):1-4. DOI: $\quad$ 10.5123/S1679$\underline{49742018000100009}$

8- Souza J, Oliveira KS, Ávila TT, Quadros SR, Zilly A, Silva-Sobrinho RA. Incidência da tuberculose e a correlação entre a realização do tratamento e a cura. Rev Enferm UFSM 2018;8(4): 637-48. DOI: 10.5902/2179769230534

9- Sousa FMP, Luna IT, Silva KL, Pinheiro PNC. Pacientes vivendo com HIV/AIDS e coinfecção tuberculose: Dificuldades associadas à adesão ou ao abandono do tratamento. Rev Gaúch Enferm. 2012;33(2):139-45. DOI: 10.1590/S198314472012000200020

10- Klopper M, Warren RM, Hayes C, Pittius NCGV, Streicher EM, Müller B, et al. Emergence and spread of extensively and totally drugresistant tuberculosis. Emerg Infect Dis. 2013;19(3): $449-55 . \quad$ DOI: 10.1590/0104070720180001710017

11- Peruhype RC, Sicsú AN, Lima MCRA, Hoffmann JF, Palha PF. Transferência de política: Perspectiva do tratamento diretamente observado da tuberculose. Texto ContextoEnferm. 2018;27(3):1-9. DOI: 10.1590/0104070720180001710017

12- Di Gennaro F, Pizzol D, Cebola B, Stubbs B, Monno L, Saracino A, et al. Social determinants of therapy failure and multi drug resistance among people with tuberculosis: A review. Tuberculosis 2017;103:44-51. DOI: 10.1016/i.tube.2017.01.002 13- Brasil, Ministério da Saúde. Programa nacional de controle da tuberculose. Brasília: Ministério da Saúde; 2004

14- Ferraz AF, Valente JG. Epidemiological aspects of pulmonar tuberculosis in Mato Grosso do Sul, Brazil. Rev Bras Epidemiol. 2014;17(1):255-66. DOI: 10.1590/1415790X201400010020ENG

15- Perrechi MCT, Ribeiro SA. Tratamento de tuberculose: Integração entre assistência e rede básica na cidade de São Paulo. J Bras Pneumol. 2009;35(11):1100-6. DOI: $10.1590 /$ S1806$\underline{37132009001100007}$

16- Reis DC, Almeida TAC, Quites HFO, Sampaio MM. Epidemiological profile of tuberculosis in the city of Belo Horizonte (MG), from 2002 to 2008. Rev Bras Epidemiol. 2013;16 (3):592-602. DOI: 10.1590/S1415-790X2013000300004

17- Salazar JLL, Asorey CCP, Arias DR, Díaz RCG, Machado LOM. Factores de riesgo socioeconómicos de la tuberculosis pulmonar en el municipio de Santiago de Cuba. Medisan 2009 [citado em 14 fev 2019]; 13(1):1-6. Disponível 
em:

http://scielo.sld.cu/scielo.php?script=sci arttext \&pid=S1029-30192009000400007

18- Shete PB, Haguma P, Miller CR, Ochom E, Ayakaka I, Davis JL, et al. Pathways and costs of care for patients with tuberculosis symptoms in rural Uganda. Int J Tuberc Lug Dis. 2015;19(8):912-7. DOI: 10.5588/ijtld.14.0166

19- Lemos LA, Feijão AR, Gir E, Galvão MTG. Aspectos da qualidade de vida de pacientes com coinfecção

20- HIV/tuberculose. Acta Paul Enferm. 2012;25(1):41-7. DOI: 10.1590/S010321002012000800007

21- Hino P, Cunha TN, Villa TCS, Santos CB. Perfil dos casos novos de tuberculose noticados em Ribeirão Preto (SP) no período de 2000 a 2006. Ciênc Saúde Coletiva 2011;16(1):1295-1301. DOI: 10.1590/S1413-81232011000700063

22- Silva DR, Muñoz-Torrico M, Duarte R, Galvão $\mathrm{T}$, Bonini EH, Arbex FF, et al. Fatores de risco para tuberculose: diabetes, tabagismo, álcool e uso de outras drogas. J Bras Pneumol. 2018;44(2):14552. DOI: $10.1590 / \mathrm{s} 1806-37562017000000443$

23- Chirinos NEC, Meirelles BHS. Fatores associados ao abandono do tratamento da tuberculose: Uma revisão integrativa. Texto Contexto-Enferm. 2011;20(3):599-606. DOI: $\underline{10.1590 / \mathrm{S} 0104-07072011000300023}$

Nota: Projeto Financiado pelo CNPQ. Chamada Universal$\mathrm{MCTI} / \mathrm{CNPq}$ № 14/2014.

Recebido em: 19/12/2018

Aprovado em: 21/11/2019

Endereço de correspondência:

Sabrina da Silva de Souza

Rua Elizeu di Bernardi 200 apto 406 A Campinas.

CEP: 88101-050- São José/SC- Brasil

E-mail: enfermeirasabrina@gmail.com 